\title{
Polarization Separation of Light Beams at the Interface of Two Mesophases"
}

\author{
A. A. Komar, M. A. Kurochkina, A. A. Melnikova, A. I. Stankevich, and A. L. Tolstik* \\ Belarusian State University, Minsk, 220030 Belarus \\ *e-mail:tolstik@bsu.by;tolstikal@mail.com \\ Received February 21, 2011
}

\begin{abstract}
The features of reflection of the linearly polarized light beams at the interface between two regions of a nematic liquid crystal (LC) with the orthogonal director orientation have been analyzed. The relationship between light beam propagation in a LC material and polarization of laser radiation has been established. Rotation of the polarization plane of a light beam has been found for the radiation introduced through the free surface "LC - air" deformed due to the capillarity effect. The total internal reflection effect has been demonstrated experimentally together with the possibility for special separation of the orthogonally polarized light beams at the interface of two mesophases.
\end{abstract}

DOI: $10.1134 / \mathrm{S} 1063785011080062$

The development and design of inexpensive compact optoelectronic devices to control the light beams is a currently one of the central trends in modern optics. Most promising electrooptical materials are liquid crystals featuring high anisotropy of the refractive index $(\sim 0.1-0.4)$ and offering its variation with the use of low applied voltage on the order of several volts. Liquid crystals are common in indicator and display systems, in manufacturing of space-time light modulators, diffraction optical elements, and the like [1-6]. The recent works devoted to the light beam propagation in spatially-structured nematic LC have demonstrated the possibility for reflection of the light beams at the interface of two LC phases $[7,8]$ and also for waveguide propagation of laser radiation $[9,10]$.

This paper presents a study into the propagation and reflection processes of light beams in thin LC layers, where the interface between two nematic mesophases is formed. The primary attention is given to analysis of the light field spatial distribution depending on polarization of laser radiation.

To form the interface of two mesophases, a LC cell has been manufactured. The cell schematic is shown in Fig. 1a. This cell comprised two glass substrates 1 with transparent electrodes of indium oxide 2 . One of the electrodes was partially etched by a photoresist applied to the glass substrate and subjected to UV irradiation through the mask covering a half of the substrate. Then the photoresist and electrode were etched step-by step. The layer of a photosensitive polymer 3 with a thickness of about $0.1 \mu \mathrm{m}$ was applied to the substrate surface by centrifugation. To impart orienting properties, the photopolymeric layer was exposed to the collimated polarized radiation of a mercury

\footnotetext{
"The article was translated by the authors.
}

lamp. Exposure time of $150 \mathrm{~s}$ for the radiation intensity $2 \mathrm{~mW} / \mathrm{cm}^{2}$ was enough to transform about $90 \%$ of the photosensitive benzaldehyde-containing component. The optical cell was filled with a liquid crystal in a vacuum chamber. Within the cell volume a planar orientation of the LC director was formed. A thickness of the LC layer was specified by spacers 4 , being $220 \mu \mathrm{m}$ for convenience of entering the radiation by the cell end. In the process of work we have used the

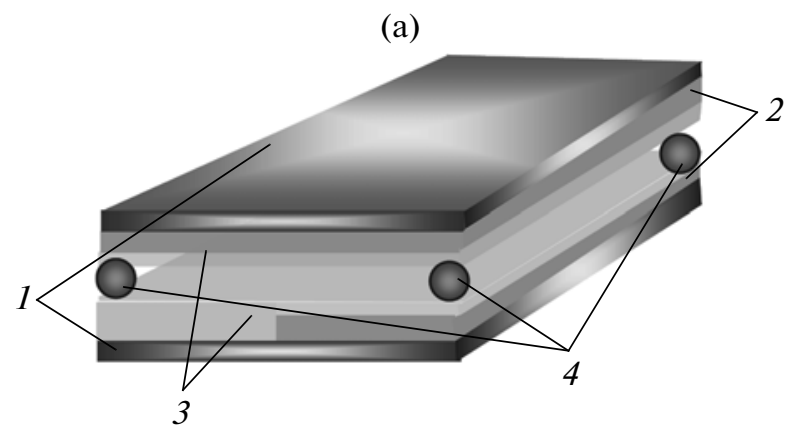

(b)

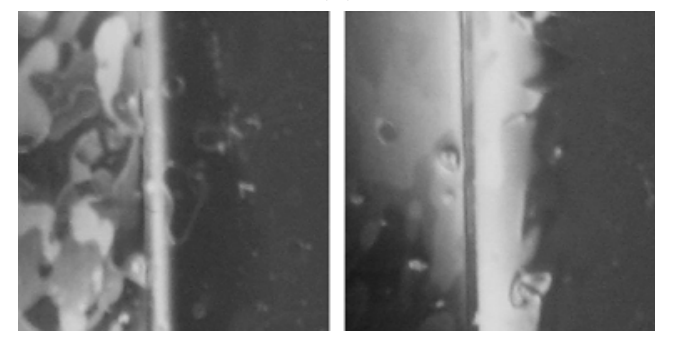

Fig. 1. (a) LC cell (see text for explanations) and (b) interface of two mesophases formed at different applied voltages: $\sim 10 \mathrm{~V}$ (left)and $\sim 50 \mathrm{~V}$ (right). 

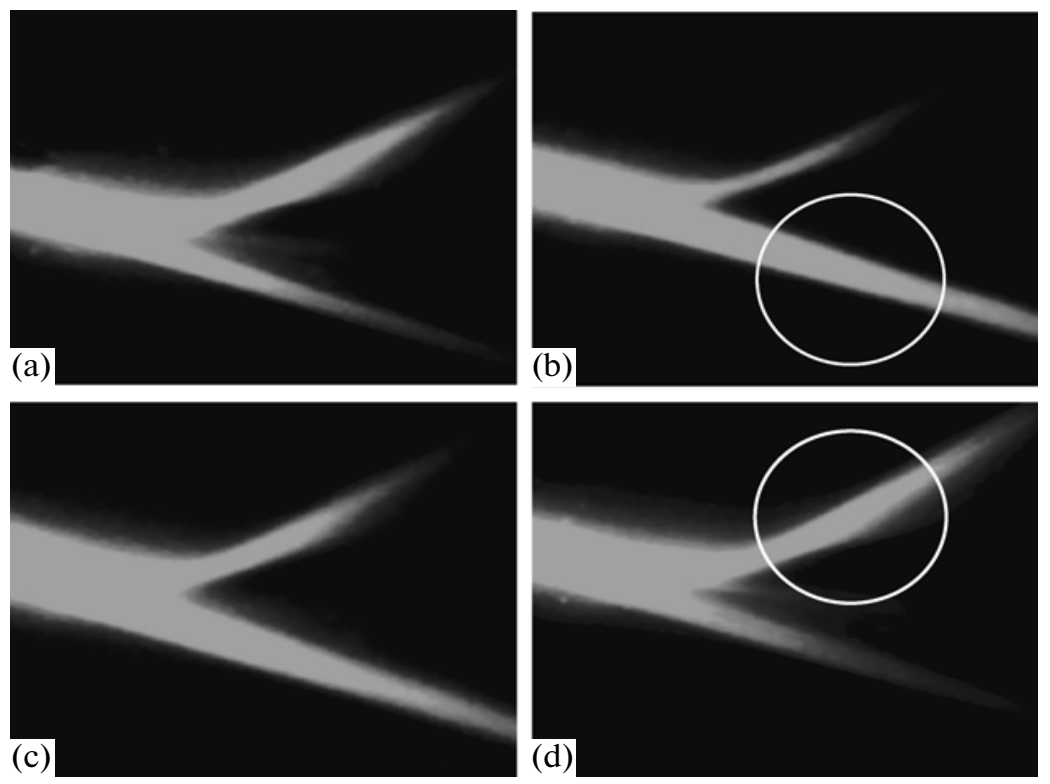

Fig. 2. Photographs of laser beam entering into the LC cell through the free surface "LC - air" for different polarizations of laser radiation: (a) $0^{\circ}$; (b) $45^{\circ}$; (c) $90^{\circ}$; (d) $135^{\circ}$.

positive birefringent nematic liquid crystal LC 1289, developed at the Research Institute for Intermediate Products and Dyes (Russia), with anisotropy of the refractive index $n_{e}-n_{0}=0.17$.

The interface was formed by application of voltage to the cell. Under the effect of an external electric field the LC molecules became reoriented precisely in the half-cell with the electrode still remaining after etching. In the other half of the cell, where the electrode was completely etched, a planar orientation of the LC molecules was retained. The regions with different orientations of the LC director (planar and homeotropic) were forming the interface between two nematic phases (Fig. 1b) with the desired dependences of refractive index on polarization of incident radiation. Visualization of the interface was realized using the crossed polarizers photography method. Dark color was associated with a planar orientation of LC. A homeotropic orientation was created in the region of the electric field application. Spatially inhomogeneous transmission of the cell in this region was caused by the incomplete LC director reorientation under the effect of an electric field due to the cell thickness as great as $220 \mu \mathrm{m}$. In crossed polarizers the twist structure formed at the interface of two LC regions appeared like a bright strip (Fig. 1b). Note that an increase in voltage was associated with widening of the near interface region. As demonstrated by the experimental studies of optical anisotropy depending on the voltage applied, despite a great thickness of the cell $(220 \mu \mathrm{m})$, considerable changes in the refractive index allowing for the formation of a pronounced interface are attained at a quite low applied voltage $(\sim 5-10 \mathrm{~V})$ meeting the external field strengths about 200-
$500 \mathrm{~V} / \mathrm{cm}$, that is lower than the strengths usually used by an order of magnitude.

Figure 2 presents the results obtained for reflection and refraction of laser beams at the interface of two mesophases. The photographs show the radiation propagation of a helium-neon laser in the LC cell depending on polarization. Entering of the radiation into the LC cell was realized at an angle providing the total internal reflection for the difference in refractive indices $\Delta n=0.7$. The electric vector orientation of a light wave entering the LC cell was taken from the light polarization direction corresponding to an ordinary wave for both regions of the cell. According to a theory, the wave with such a polarization should not be reflected from the interface. Light having the perpendicular polarization is associated with the angle $90^{\circ}$. In this case the wave is an ordinary wave for the planar orientation of LC and an extraordinary wave for the homeotropic orientation. Because of this, the total reflection effect must be observed at the interface. But during the experiment in both cases $\left(0^{\circ}\right.$ and $\left.90^{\circ}\right)$ one could observe two beams, transmitted and reflected (Figs. 2a, 2c), despite the fact that the incidence angle of a laser beam on the interface was greater than the total internal reflection angle. At the same time, reflection was at a minimum for the angles close to $130^{\circ}$ (Fig. 2d) and at a maximum at $45^{\circ}$ (Fig. 2b). This situation was attributed by the authors to a change in polarization of laser radiation when the radiation was entered into the cell through the free surface "LCair" that was concave in form due to the capillarity effects. At the entrance point into the cell at the interface "LC - air" the formed twist-structure rotates the 

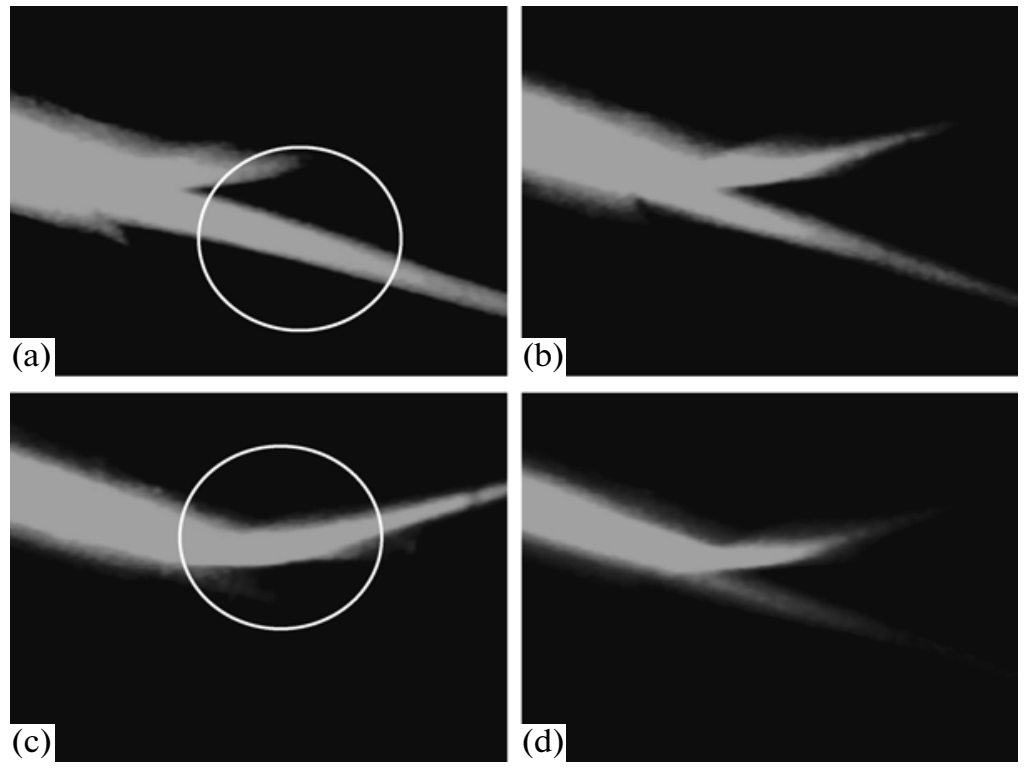

Fig. 3. Photographs of a laser beams entering into the LC cell through the end glass plate for different polarizations of laser radiation: (a) $0^{\circ}$; (b) $45^{\circ}$; (c) $90^{\circ}$; (d) $135^{\circ}$.

radiation polarization vector by an angle approximating $45^{\circ}$.

To verify the assumption that the radiation polarization plane is rotated at the entrance into the cell, an LC element with a glass plate glued at the end (on the entrance side) was manufactured. The plate was covered with the aligning coating that was preliminary rubbed in accordance with a homeotropic orientation of the LC director in the cell. The experimental results are given in Fig. 3. It is seen that due to entering of the radiation through the glass plate, there is no effect associated with the polarization plane rotation of laser radiation. As expected, the total internal reflection was observed at the angle $90^{\circ}$ (Fig. 3c) associated with a vertical polarization of radiation. In the case of a horizontal polarization (angle of $0^{\circ}$ ), the light was propagating in the cell practically without reflection (Fig. 3a).

In this way the conducted experimental studies have demonstrated the possibility for reflection of laser beams at the interface between two mesophases and have enabled optimization of the conditions for the total internal reflection with regard to the revealed effect of the polarization plane rotation of a light beam when entered through the free surface "LC-air". The use of the relationship between refractive index and polarization of laser radiation looks most promising for the realization in practice. Using this relationship, one can realize separation of the light beams with orthogonal polarizations, e.g., TE and TM waveguide modes, and can develop a compact LC polarizer.
This work was supported by the Belarusian Foundation for Fundamental Research, Project F10MC02 .

\section{REFERENCES}

1. T. Matsui, M. Ozaki, and K. Yoshino, J. Opt. Soc. Am. B 21, 1651 (2004).

2. P. Pagliusi and G. Gipparrone, J. Opt. Soc. Am. B 21, 996 (2004).

3. T.-H. Lin and A. Y.-G. Fuh, Opt. Lett. 30, 13901 (2005).

4. L. Song and W.-K. Lee, Opt. Commun. 259, 293 (2006).

5. O. Trushkevych, N. Collings, W. A. Crossland, and T. D. Wilkinson, Appl. Opt. 45, 8889 (2006).

6. A. A. Kazak, E. A. Melnikova, A. L. Tolstik, U. V. Mahilny, and A. I. Stankevich., Pis'ma Zh. Tehh. Fiz. 34 (10), 1 (2008) [Tech. Phys. Lett. 34, 861 (2008)].

7. A. G. Maksimochkin, S. V. Pasechnik, V. A. Tsvetkov, D. A. Yakovlev, G. I. Maksimochkin, and V. G. Chigrinov, Opt. Commun. 270, 273 (2007).

8. M. Peccianti, A. Dyadyusha, M. Kaczmarek, and G. Assanto, Nature Phys. 2, 737 (2006).

9. M. Peccianti, C. Conti, and G. Assanto, Opt. Lett. 28, 2231 (2003).

10. A. G. Maksimochkin, S. V. Pasechnik, G. I. Maksimochkin, and V. G. Chigrinov, Opt. Commun. 283, 3136 (2010). 